\title{
Host-guest chemistry with water-soluble gold nanoparticle supraspheres
}

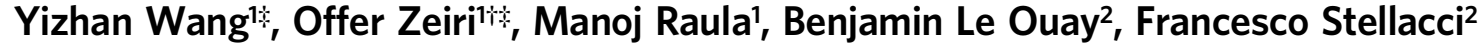 \\ and Ira A. Weinstock ${ }^{1 \star}$
}

\begin{abstract}
The uptake of molecular guests, a hallmark of the supramolecular chemistry of cages and containers, has yet to be documented for soluble assemblies of metal nanoparticles. Here we demonstrate that gold nanoparticle-based supraspheres serve as a host for the hydrophobic uptake, transport and subsequent release of over two million organic guests, exceeding by five orders of magnitude the capacities of individual supramolecular cages or containers and rivalling those of zeolites and metal-organic frameworks on a mass-per-volume basis. The supraspheres are prepared in water by adding hexanethiol to polyoxometalate-protected $4 \mathrm{~nm}$ gold nanoparticles. Each $200 \mathrm{~nm}$ assembly contains hydrophobic cavities between the estimated 27,400 gold building blocks that are connected to one another by nanometre-sized pores. This gives a percolated network that effectively absorbs large numbers of molecules from water, including 600,000, 2,100,000 and 2,600,000 molecules (35, 190 and $234 \mathrm{~g} \mathrm{I}^{-1}$ ) of para-dichorobenzene, bisphenol A and trinitrotoluene, respectively.
\end{abstract}

$\mathrm{H}$ ost-guest phenomena that involve the uptake of gases and small molecules are associated with the supramolecular chemistry of soluble capsules, cages and containers $^{1-5}$ or, alternatively, with heterogeneous reactions of porous solid-state materials such as zeolites ${ }^{6}$ and metal-organic frameworks ${ }^{7}$. Only now, however, are organized assemblies of metal nanoparticles beginning to serve in a similar capacity as hosts for molecular guests. In organic solvents, for example, light-induced dipoledipole interactions between gold nanoparticles with azobenzenefunctionalized thiolate ligands were recently used to entrap and catalyse the reactions of polar and alkylaromatic substrates ${ }^{8}$. By design, substrates were entrapped during nanoparticle aggregation to overcome the poor uptake and diffusion of molecular guests into colloidal nanocrystal assemblies. However, the uptake of molecular guests, a trait of supramolecular and solid-state chemistry, has so far not been achieved for colloidal nanoparticle assemblies.

For that to occur, a thermodynamically favourable driving force must be combined with a porous structure whose interior architecture features host cavities, along with pathways for the effective diffusion of molecular guests from the exterior (bulk solution) to host domains deeply buried in the assembly's interior. If this were achieved, colloidal metal-nanoparticle assemblies could emerge as a new class of functional nano-engineered structures that are uniquely positioned between supramolecular containers and porous solid-state materials.

We report a new class of functional assemblies, wherein the hydrophobic effect ${ }^{9,10}$ drives the spontaneous uptake of alkyl and alkylaromatic guests ${ }^{11}$ by porous $200 \mathrm{~nm}$ diameter supraspheres whose host capacities are orders of magnitude larger than those of individual cages or containers. On a mass-per-volume basis, the level of uptake rivals those of zeolites and metal-organic frameworks, which, for methane at elevated pressures, reach values of 52 and $160 \mathrm{~g} \mathrm{l}^{-1}$, respectively, for a crystalline aluminosilicate (zeolite) with $5 \AA$ pores (100 bar, $293 \mathrm{~K})^{12}$ and the metal-organic framework, Fe(bdp), comprising Fe(II) and 1,4-benzenedipyrazolate linkages $(70 \text { bar, } 298 \mathrm{~K})^{13}$.

The uptake of truly large numbers of hydrophobic guests is a natural consequence of moving from molecular to nanoscale building units. Namely, instead of individual supramolecular cages or containers, the use of hydrocarbon-coated gold nanoparticles as building units leads to large-capacity multi-container assemblies. Moreover, the size of the hydrophobic (host) domains in the supraspheres (a geometric function of the radius of the gold nanoparticles), is larger than those found in supramolecular cages or metal-organic frameworks. We demonstrate the unique properties of the suprasphere hosts by using them to concentrate (adsorb), separate and subsequently release over two million hydrophobic guests. We additionally show how the use of polyoxometalate leaving groups provides options for tuning the properties of the suprasphere's outer surfaces for chemoselective absorption of targeted hydrophobic analytes.

\section{Hydrophobically driven assembly in water}

Although a variety of gold nanoparticle (Au NP) supraspheres have been prepared ${ }^{14-17}$, the hydrophobically driven formation of analogous assemblies in pure water remains elusive ${ }^{18-21}$. The main obstacle is that precipitation rapidly results from a lack of control over the aggregation processes ${ }^{19}$. This was overcome by first placing the highly negatively charged $1 \mathrm{~nm}$ diameter cluster anion, $\mathrm{AlW}_{11} \mathrm{O}_{39}^{9-}$ (1; a polyoxometalate $)^{22}$ as versatile leaving groups on the surfaces of $4 \mathrm{~nm}$ diameter Au NPs. The self-assembly is then driven by simply adding hexanethiol (hex-SH) to solutions of the 1-protected Au NPs. Central to the success of this approach is the unique kinetic and thermodynamic properties of 1 monolayers on $\mathrm{Au}$ NPs in water ${ }^{23-25} .1$ is a much better protecting ligand than citrate anion, but is less strongly bound to gold surfaces than are thiols. As a result, the hydrophilic polyoxometalate monolayer directs the selective placement of thiolate ligands into discrete hydrophobic domains on the $\mathrm{Au} \mathrm{NPs}{ }^{26,27}$, while intact domains of 1 remain in place to continuously impart solubility in water. As more alkanethiol is added, the 1 domains are gradually displaced, leading to the controlled hydrophobic assembly of supraspherical clusters ${ }^{18}$.

Namely, as aliquots of aqueous hex-SH were added incrementally to solutions of 1-protected Au NPs (Fig. 1a), the surface plasmon resonance (SPR) absorbance at $750 \mathrm{~nm}$ (indicative of aggregation)

'Department of Chemistry and the Ilse Katz Institute for Nanoscale Science \& Technology, Ben-Gurion University of the Negev, Beer Sheva 84105, Israel. ${ }^{2}$ Institute of Materials, École Polytechnique Fédérale de Lausanne (EPFL), Lausanne 1015, Switzerland. 'Present address: Nuclear Research Center Negev, Beer-Sheva 84190, Israel. *These authors contributed equally to this work. *e-mail: iraw@bgu.ac.il 

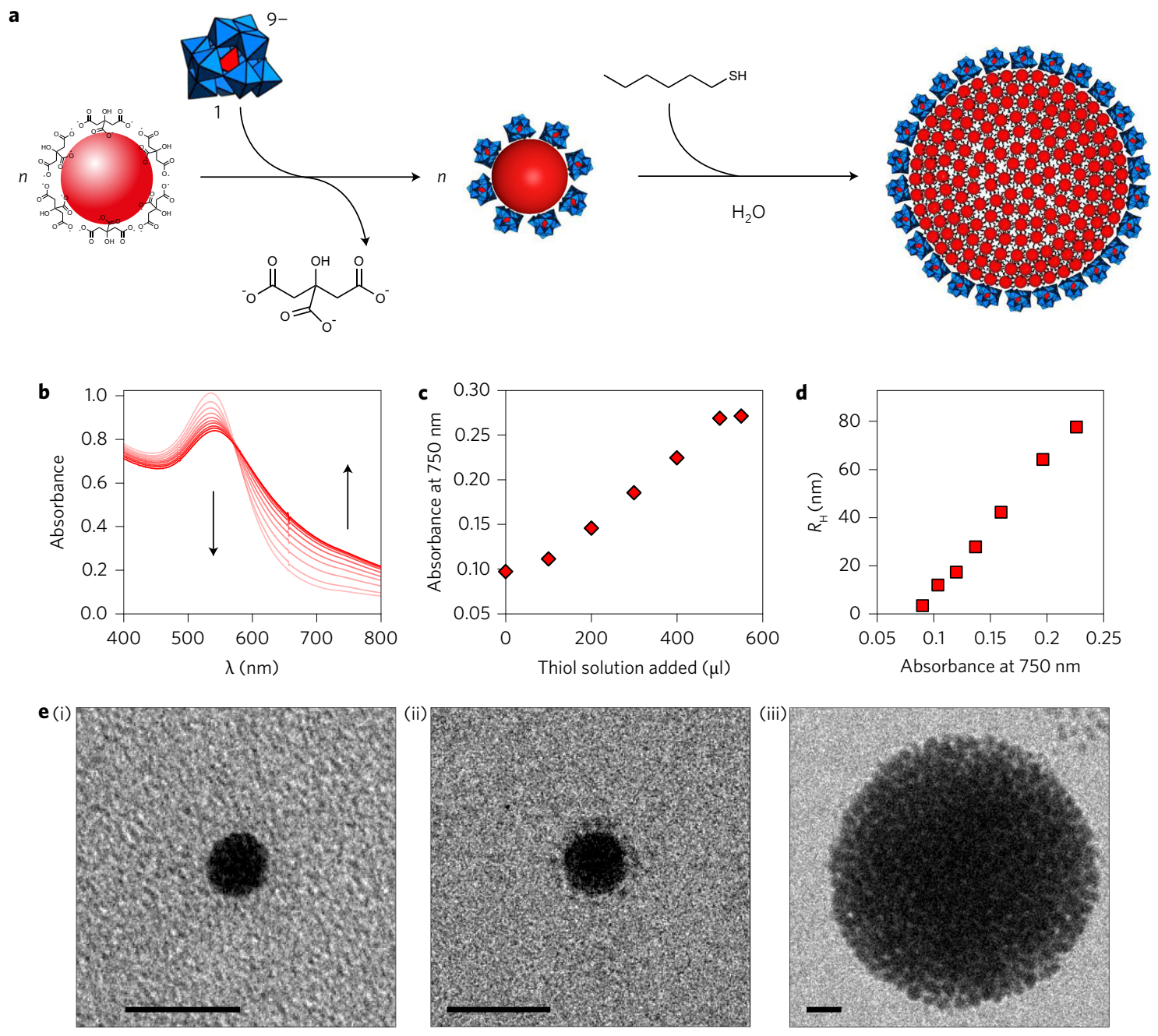

Figure 1 | Reaction of hex-SH with 1-protected $\mathbf{4} \mathbf{n m}$ diameter Au NPs in water. a, Schematic showing how citrate ligands are replaced by 1, to give 1-protected Au NPs. A reaction with hex-SH then leads to the formation of colloidal supraspheres. The structure of 1 is shown in polyhedral notation: $\mathrm{W}(\mathrm{VI}$ )-centred polyhedra are in blue (oxygen atoms are present at all vertices) and the centrally located, four-coordinate $\mathrm{Al}(\mathrm{III})$ ion is in red. $\mathbf{b}$, Changes of the SPR absorbance with incremental additions of aqueous hex-SH (saturated solution). Arrows show the directions of change in absorbance, with the increase at $750 \mathrm{~nm}$ indicative of Au NP aggregation. c, Absorbance at $750 \mathrm{~nm}$ as a function of hex-SH addition. The plateau at around 0.27 absorbance units is approached as nearly all the molecules of 1 have been displaced from the Au NP surfaces. $\mathbf{d}$, Average hydrodynamic radii (from DLS measurements; Supplementary Fig. 1) as a function of absorbance at $750 \mathrm{~nm}$ before reaching the plateau value in c. (Initial absorbance values varied by \pm 0.02 units for different samples of 1-protected Au NPs) e, Cryo-TEM images illustrating colloidal suprasphere formation: (i) and (ii) show individual citrate- and 1-protected Au NPs, respectively; (iii) shows a representative intermediate-sized colloidal suprasphere, with a radius of around $45 \mathrm{~nm}$. Scale bar, $10 \mathrm{~nm}$.

increased, eventually reaching a plateau (Fig. 1b,c). The onset of this plateau indicated that the hydrophobically driven self-assembly had reached completion. Before this plateau value, dynamic light scattering (DLS) data revealed a linear correlation between the average hydrodynamic radii, $R_{\mathrm{H}}$, of the assemblies, and the increase in absorbance at $750 \mathrm{~nm}$ (Fig. 1d and Supplementary Fig. 1).

This correlation was used to reproducibly prepare colloidal assemblies containing tens of thousands of hexanethiolate (hex-S) protected Au NPs, and the spectroscopically monitored growth process can be used to prepare supraparticles of specific sizes. The fine control over Au NP self-assembly in water is attributed to the influence of the 1 ligand shells: in the absence of 1 (for example, when a citrate anion is used as a protecting ligand) the incremental addition of hex-SH rapidly results in complete precipitation of the Au NPs. The overall process is documented in Fig. 1e, in which cryogenic transmission electron microscopy (cryo-TEM) images show each of the structures illustrated in Fig. 1a.

Additional cryo-TEM imaging revealed intermediate structures present during the assembly process. As hex-SH is added, 1-protected
$\mathrm{Au}$ NPs are first converted into dimers and tetramers (Fig. 2a,b), and then into small clusters with radii of from 10 to $20 \mathrm{~nm}$ (Fig. 2c). These clusters are held together by hydrophobic hex-S ligand-shell domains ${ }^{26,28}$, but remain soluble in water due to the presence of intact hydrophilic domains of $1^{27}$. As more hex-SH is added, fewer individual Au NPs are observed by DLS (Supplementary Fig. 1) and the radii of the clusters increase smoothly, leading to soluble (colloidal) supraspheres (Figs 1e, 2d and Supplementary Fig. 2). (If more hex-SH is added, particles with diameters of approximately $200 \mathrm{~nm}$ are obtained, but must be stabilized by the addition of capping ligand; see below.)

The spherical shape of the colloidal assemblies was confirmed in a statistically definitive DLS experiment (Fig. 2e) in which $R_{\mathrm{H}}$ values of a solution of intermediate-sized supraspheres remained constant as the detector angle was varied from $30^{\circ}$ to $150^{\circ}$. Consistent with this, the imaged diameter of the assembly in Fig. $2 \mathrm{~d}$ remained constant at $99 \pm 2 \mathrm{~nm}$ on tilting of the specimen grid over a range of nearly $90^{\circ}$ (inset to Fig. 2e). 

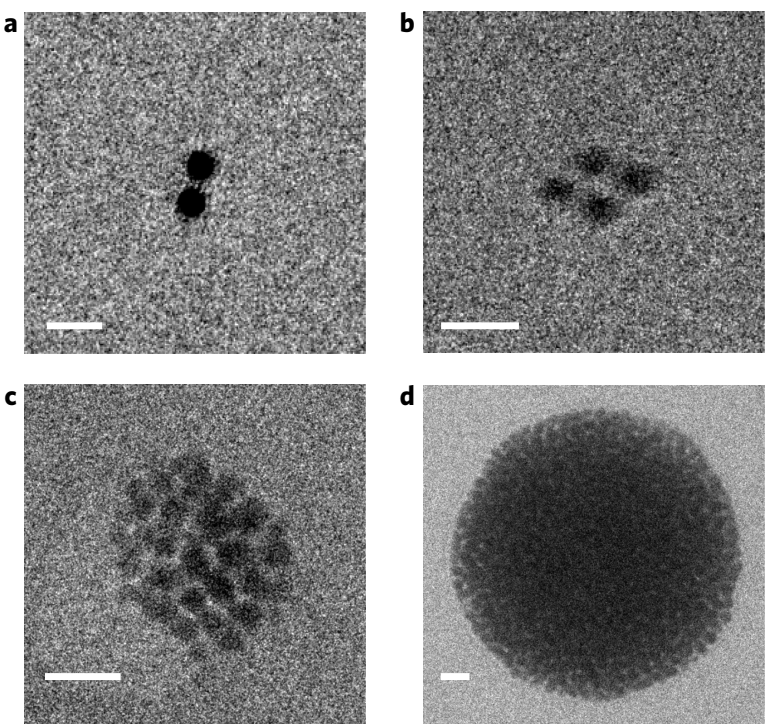

e

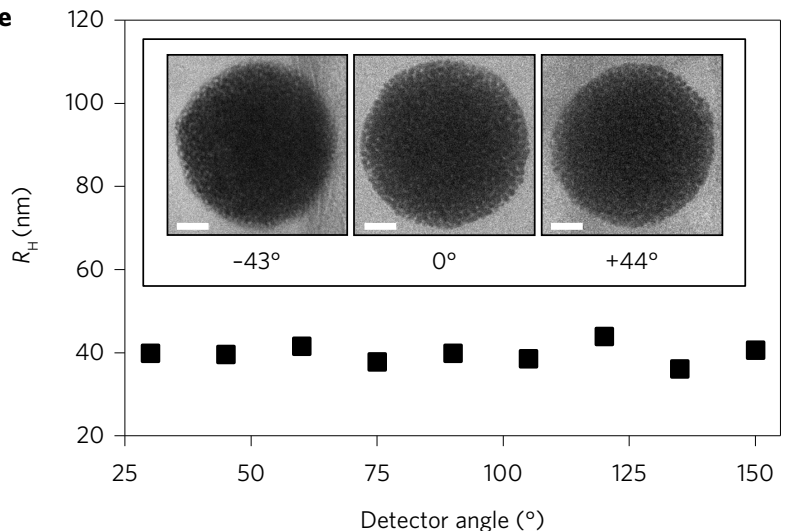

Figure 2 | Cryo-TEM images and DLS data documenting the growth and shape of colloidal supraspheres. a-c, A dimer (a), a tetramer (b) and a larger-nuclearity cluster present early in the assembly (c). d, A colloidal suprasphere. e, In the variable-angle DLS study, the average hydrodynamic radius of a solution of intermediate-sized colloidal supraspheres remained constant as the detector angle was varied from $30^{\circ}$ to $150^{\circ}$. Inset: cryo-TEM images of the assembly shown in $\mathbf{d}$ were obtained at tilt angles of $-43^{\circ}$ to $+44^{\circ}$ relative to the electron beam. Consistent with the spherical shape indicated by the DLS data, tilting had no effect on the imaged diameter of the assembly, which remained constant at $99 \pm 2 \mathrm{~nm}$. Scale bar, $10 \mathrm{~nm}$ (a-d); $20 \mathrm{~nm}$ (e, inset).

Initially formed supraspheres of up to ca. $150 \mathrm{~nm}$ in diameter are soluble and stable in water for several weeks, with solubility in water that is attributed to hydrophilic domains of 1 (and its countercations) on $\mathrm{Au} \mathrm{NPs}$ at the suprasphere-water interface. (Direct evidence for these hydrophilic domains is provided as Supplementary Information; see Supplementary Figs 3-5). For large supraspheres with diameters of $200 \mathrm{~nm}$, however, the polyoxometalate domains are less able to impart stability for long periods of time, and the clusters precipitate from solution within $24 \mathrm{~h}$. This can be entirely prevented, however, if the polyoxometalates that remain at the cluster-water interface are replaced by water-soluble thiolate capping ligands.

Moreover, the simple introduction of capping ligands provides for a degree of modifiability that is highly atypical of Au NP assemblies, which can be used in the systematic variation of suprasphere charges from negative to positive, or to neutral, for example. This was done by capping freshly prepared $200 \mathrm{~nm}$ supraspheres with $\mathrm{HS}\left(\mathrm{CH}_{2}\right)_{10} \mathrm{CO}_{2}^{-}\left(\mathrm{HSR}^{-}\right)$, and $\mathrm{HS}\left(\mathrm{CH}_{2}\right)_{11} \mathrm{~N}\left(\mathrm{CH}_{3}\right)_{3}{ }^{+}\left(\mathrm{HSR}^{+}\right)$or
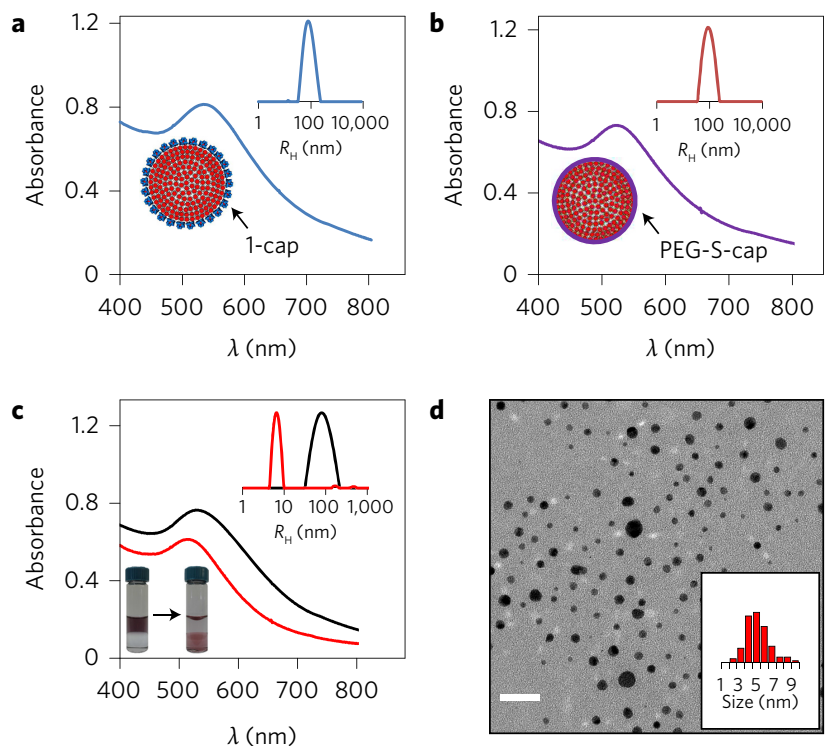

Figure 3 | PEG-S capping of 1-protected colloidal supraspheres in water and their disassembly on phase transfer to $\mathrm{CH}_{2} \mathrm{Cl}_{2}$. a, Ultraviolet-visible spectrum and DLS profile (inset) of a solution of freshly prepared $200 \pm 25 \mathrm{~nm}$ diameter colloidal supraspheres, capped by monolayer domains of 1 on the outermost Au NPs. b. Ultraviolet-visible spectrum and DLS profile (inset) of highly stable supraspheres after replacing 1 by PEG-S in water. c, Ultraviolet-visible spectra and DLS profiles (upper inset) before and after phase transfer from water (black curves) to $\mathrm{CH}_{2} \mathrm{Cl}_{2}$ (red curves), respectively. Lower inset: photographs of PEG-S-capped supraspheres in water (top layer; purple), and after phase transfer and disassembly in $\mathrm{CH}_{2} \mathrm{Cl}_{2}$ (bottom layer; red). d, TEM image of PEG-S-capped supraspheres after their disassembly in $\mathrm{CH}_{2} \mathrm{Cl}_{2}$. Inset: size distribution of the hex-S-capped Au NPs. Scale bar, $20 \mathrm{~nm}$.

mercapto-polyethylene glycol, PEG-SH (molecular weight = 2,015 Da), (Fig. 3a,b and Supplementary Figs 6 and 7). Notably, the addition of the capping ligands caused no significant changes in absorbance at $750 \mathrm{~nm}$ or in $R_{\mathrm{H}}$ values as determined by DLS.

Once capped, the supraspheres are stable for at least six months at room temperature, and can be centrifuged to a dense pellet and re-dispersed with no changes in the ultraviolet-visible spectra or $R_{\mathrm{H}}$ (DLS). Owing to their large mass and density, the thiolate-capped assemblies diffuse slowly towards the bottom of storage vials, giving dark solutions that gradually become colourless towards the tops of the liquid samples. However, the process is entirely reversible: gentle agitation immediately regenerates a clear purple solution, with no precipitate and no changes in the ultraviolet-visible spectra or $R_{\mathrm{H}}$.

Importantly, the neutrally charged PEG-S capping ligands are soluble in both water and methylene chloride $\left(\mathrm{CH}_{2} \mathrm{Cl}_{2}\right)$. This solubility behaviour was used to confirm that the supraspheres in fact comprised individual hex-S-capped $\mathrm{Au}$ NPs. Following phase transfer of the purple-coloured PEG-S-capped supraspheres into $\mathrm{CH}_{2} \mathrm{Cl}_{2}$, the hydrophobic driving force for assembly in water was lost and dissociation into individual hex-S-capped $\mathrm{Au}$ NPs was indicated by a red-coloured $\mathrm{CH}_{2} \mathrm{Cl}_{2}$ solution (the ultravioletvisible absorbance at $750 \mathrm{~nm}$ decreased dramatically; Fig. 3c). Dissociation into individual Au NPs was confirmed by DLS (inset to Fig. $3 c$ and Supplementary Figs 8 and 9) and TEM images of dried samples (Fig. 3d).

\section{Uptake and release of molecular organic guests}

The transition from hydrophobically stabilized clusters in water to complete disassembly in $\mathrm{CH}_{2} \mathrm{Cl}_{2}$ was used to quantitatively investigate the $200 \mathrm{~nm}$ diameter PEG-S-capped supraspheres as 

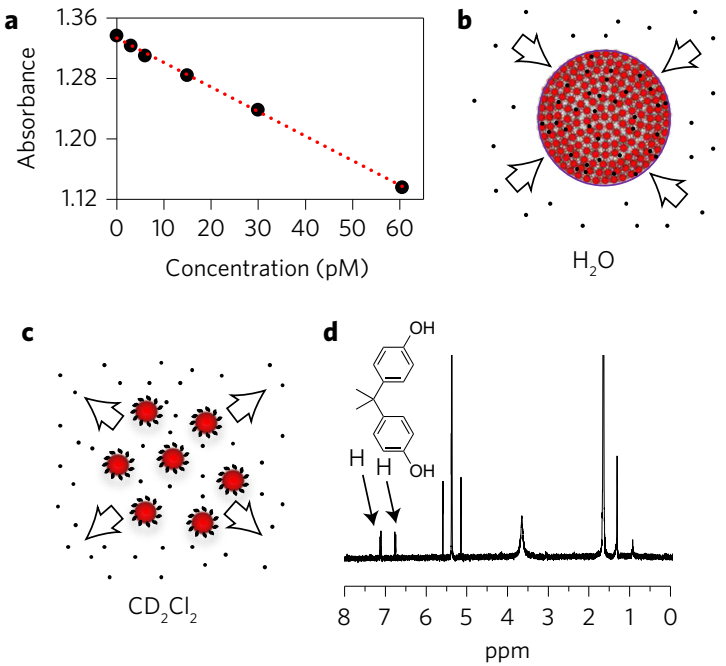

Figure 4 | Uptake and release of hydrophobic guests by colloidal supraspheres. a, Decrease in absorbance of BPA $(1.5 \mathrm{ml}, 0.85 \mathrm{mM})$ in water after uptake by increasingly larger concentrations of PEG-S-capped $200 \pm 25 \mathrm{~nm}$ diameter colloidal supraspheres. (Control experiments, included in Supplementary Fig. 10, ruled out adsorption of BPA within the PEG-S capping ligand shells.) After $24 \mathrm{~h}$ at room temperature, the supraspheres were removed by centrifugation (10 $\mathrm{min}$ at 13,500 r.p.m.) and the $278 \mathrm{~nm}$ absorbance of BPA in the supernatant solution was measured by ultraviolet-visible spectroscopy. $\mathbf{b}$, The decrease in absorbance is due to the uptake process indicated, while the linear relationship in $\mathbf{a}\left(R^{2}=0.999\right)$ indicates that each similarly sized suprasphere serves as a host for equal numbers of BPA guests. DLS measurements before and after uptake revealed no change in the $200 \pm 25 \mathrm{~nm}$ diameter of the PEG-S-capped supraspheres (Supplementary Fig. 11). c, The subsequent release of BPA guests on dissolution of the isolated (and washed) colloidal supraspheres in $\mathrm{CD}_{2} \mathrm{Cl}_{2}$. $\mathbf{d}$, The BPA guests released into $\mathrm{CD}_{2} \mathrm{Cl}_{2}$ were readily observed and quantified by ${ }^{1} \mathrm{H}$ NMR spectroscopy. The spectrum was obtained after BPA uptake by a $30 \mathrm{pM}$ solution of supraspheres.

supraparticle hosts for the uptake and release of organic guests such as bisphenol A (BPA, Fig. 4). BPA is sparingly soluble in water $(1.3 \mathrm{mM}$ at $293 \mathrm{~K})$, and well known for its use in the manufacture of polycarbonate-based plastics. It was chosen here because it provides both strong absorbance in the ultraviolet region, for spectroscopically quantifying its uptake, and two sets of chemically equivalent aromatic protons for analysis by ${ }^{1} \mathrm{H}$ NMR spectroscopy after release into $\mathrm{CH}_{2} \mathrm{Cl}_{2}$. The uptake experiments were carried out by first quantifying the removal of BPA $(0.85 \mathrm{mM})$ from water as a function of the concentration of PEG-S-capped supraspheres (varied from 0 to $61 \mathrm{pM}$ ). After $24 \mathrm{~h}$ at room temperature, the supraspheres were removed by centrifugation and the $278 \mathrm{~nm}$ absorbance maximum of BPA in the colourless supernatant solutions was quantified by ultraviolet-visible spectroscopy (Fig. 4a and Supplementary Fig. 10). The linear decrease in absorbance demonstrated that each suprasphere serves as a host for the same number of BPA guests (the uptake process is illustrated in Fig. $4 \mathrm{~b}$ ).

The data in Fig. 4a further indicated that each suprasphere assembly hosts a very large number of the molecular guests. The average concentration of BPA in each assembly was $0.8 \pm 0.1 \mathrm{M}$, corresponding to $2.1 \pm 0.3$ million guests per suprasphere host (see calculations in Supplementary Information). To help visualize this large number of guests, it corresponds to 77 equivalents of BPA for each of the assembly's approximately 27,400 Au NPs. Given the 'footprint' (surface area) occupied by alkanethiolate ligands on the Au NP surface ${ }^{27}$, each $4 \mathrm{~nm}$ diameter gold core is surrounded by an estimated 240 hex-S ligands, giving a ratio of one BPA guest to every three hex-S ligands within the host assembly.
This 1:3 ratio was independently confirmed by comparing the relative intensities of signals from BPA to those of hex-S ligands in the ${ }^{1} \mathrm{H}$ NMR spectrum obtained after guest 'release' in $\mathrm{CD}_{2} \mathrm{Cl}_{2}$ (Fig. 4d and Supplementary Figs 12 and 13). The released guests were clearly observed by ${ }^{1} \mathrm{H}$ NMR spectroscopy (Fig. $4 \mathrm{~d}$; signals due to the aliphatic protons are obscured by signals from residual water at $1.64 \mathrm{ppm})$. Integration of the signal intensities gave the same 1:3 ratio of guests to hex-S ligands as that obtained by ultraviolet-visible spectroscopy using the data in Fig. 4a.

To establish the generality and better define the scope of the host-guest chemistry, we explored the uptake of several hydrophobic guests: a dye (azulene), two explosives (2,4,6-trinitrotoluene (TNT) and 1,3,5-trinitroperhydro-1,3,5-triazine (RDX)), a common herbicide (Alachlor) and the alkyl- and (toxic) halogenatedaromatic compounds, para-xylene and para-dichlorobenzene ( $p$-DCB), (structural drawings are provided in Table 1).

As with BPA (entry 1), the spectrophotometric properties of azulene (entry 2) were used to quantify its uptake from water (Table 1). Here, its fluorescence-band intensity decreased linearly with increasing concentrations of added PEG-S-capped supraspheres. The results (see Supplementary Figs 14 and 15) were statistically identical to those obtained for BPA (Fig. 4a), with $2.5 \pm 0.2$ million azulene guests sequestered by each colloidal suprasphere.

For the other compounds listed in Table 1, uptake was quantified by ${ }^{1} \mathrm{H}$ NMR spectroscopy after isolation and disassembly in $\mathrm{CD}_{2} \mathrm{Cl}_{2}$, as discussed above for BPA (Supplementary Figs 16-22). In initial reactions with TNT (entry 3), uptake into the host assemblies was not observed. We suspect this is a kinetic issue, related to slow diffusion through the $10 \mathrm{~nm}$ thick polyethylene glycol shell of the PEG-S capping ligands. To address this, analogous $200 \mathrm{~nm}$ diameter assemblies were capped by $50 \%$ PEG-S and $50 \%$ hex-S protecting ligands to introduce hydrophobic channels for more kinetically facile transport of guests into the interior of the supraspheres (see Methods and Supplementary Information). Using these mixedcapping-ligand assemblies, ${ }^{1} \mathrm{H}$ NMR indicated the uptake of $2.6 \pm 0.6$ million TNT or RDX guests (entries 3 and 4), which is statistically identical to the values obtained for BPA and azulene. Using the same mixed capping ligands, ${ }^{1} \mathrm{H}$ NMR indicated an uptake of 1.3 million Alachlor guests, 600,000 molecules of $p$-DCB and 1.6 million molecules of $p$-xylene (entries 5-7).

\section{A percolated network of hydrophobic holes}

The large numbers of hydrophobic guests reported in Table 1, in several cases exceeding two million per suprasphere (Fig. 5a), can be explained by the presence of the void space in (ideally) closestpacked-both cubic and hexagonal-assemblies of identical spheres $^{29}$. In both closest-packed phases, $N$ spheres occupy $74 \%$ of the total volume, leaving $26 \%$ of the volume as void space. This empty space comprises $N$ octahedral $\left(O_{\mathrm{h}}\right)$ holes (Fig. $\left.5 \mathrm{~b}\right)$ and $2 N$ tetrahedral $\left(T_{\mathrm{d}}\right)$ holes.

As is typical for preparations of small Au NPs in water, the $4 \mathrm{~nm}$ (average) diameter metallic cores of the nanoparticles in the supraspheres possess a degree of polydispersity, thus deviating slightly from the perfect size uniformity required to obtain crystalline phases. As such, although data from small angle X-ray scattering of the $200 \mathrm{~nm}$ diameter supraspheres (Supplementary Fig. 23) do not support the formation of cubic or hexagonal phases, they do reveal an average repeating (interparticle) distance of $6.0 \pm 0.1 \mathrm{~nm}-$ as expected for tightly packed hexanethiolate-capped $4 \mathrm{~nm}$ diameter core Au NPs. Importantly, however, the $26 \%$ void space in closest-packed structures places a lower limit on the actual void space. Hence, the total void space formed by approximately $O_{\mathrm{h}}$ and $T_{\mathrm{d}}$ cavities must be at least $26 \%$ of the volume of each supraspherical assembly.

The host-guest chemistry was examined in detail by considering the occupancies of the (idealized) $O_{\mathrm{h}^{-}}$and $T_{\mathrm{d}}$-symmetry cavities. 
Table 1 | Uptake of hydrophobic guests by $200 \mathrm{~nm}$ diameter colloidal supraspheres ${ }^{\star}$.

\begin{tabular}{|c|c|c|c|c|c|c|c|}
\hline Entry & Substrate & Chemical structure & $\begin{array}{l}\text { Ligand-shell } \\
\text { composition }\end{array}$ & $\begin{array}{l}\text { Guest concentration } \\
\text { in each assembly }(M)\end{array}$ & $\begin{array}{l}\text { Numbers of } \\
\text { guests per } \\
\text { assembly }\left(\times 10^{6}\right)\end{array}$ & $\begin{array}{l}\text { van der Waals } \\
\text { volume of each } \\
\text { guest }\left(\AA^{3}\right)\end{array}$ & $\begin{array}{l}\text { Guest- } \\
\text { occupied void } \\
\text { space (\%) }\end{array}$ \\
\hline 1 & $\mathrm{BPA}^{\dagger}$ & & PEG-S & $0.8 \pm 0.1$ & $2.1 \pm 0.3$ & 223 & $43 \pm 6$ \\
\hline 2 & Azulene & & PEG-S & $0.9 \pm 0.1$ & $2.5 \pm 0.2$ & 122 & $28 \pm 2$ \\
\hline 3 & $\mathrm{TNT}^{\varsigma}$ & & $\begin{array}{l}\text { 50:50 } \\
\text { PEG-S:hex-S }\end{array}$ & $1.0 \pm 0.2$ & $2.6 \pm 0.6$ & 176 & $42 \pm 10$ \\
\hline 4 & $\mathrm{RDX}^{\varsigma}$ & & $\begin{array}{l}\text { 50:50 } \\
\text { PEG-S:hex-S }\end{array}$ & $1.0 \pm 0.2$ & $2.6 \pm 0.6$ & 159 & $38 \pm 9$ \\
\hline 5 & Alachlor $^{\varsigma}$ & & $\begin{array}{l}\text { 50:50 } \\
\text { PEG-S:hex-S }\end{array}$ & $0.5 \pm 0.1$ & $1.3 \pm 0.3$ & 261 & $31 \pm 7$ \\
\hline 6 & $p-\mathrm{DCB}^{\S}$ & & $\begin{array}{l}\text { 50:50 } \\
\text { PEG-S:hex-S }\end{array}$ & $0.2 \pm 0.1$ & $0.6 \pm 0.2$ & 112 & $6 \pm 2$ \\
\hline 7 & $p$-xylene" & & $\begin{array}{l}\text { 50:50 } \\
\text { PEG-S:hex-S }\end{array}$ & $0.6 \pm 0.1$ & $1.6 \pm 0.3$ & 116 & $17 \pm 3$ \\
\hline 8 & $\begin{array}{l}\text { BPA and } \\
\text { TNT }^{s}\end{array}$ & As above & $\begin{array}{l}\text { 50:50 } \\
\text { PEG-S:hex-S }\end{array}$ & $\begin{array}{l}0.4 \pm 0.1(B P A) \\
0.6 \pm 0.1(T N T)\end{array}$ & $2.3 \pm 0.5$ (sum) & as above & $40 \pm 9$ (sum) \\
\hline 9 & $\begin{array}{l}\text { BPA and } \\
\text { TNT }^{\oplus}\end{array}$ & As above & PEG-S & $0.7 \pm 0.2(\mathrm{BPA})$ & $1.9 \pm 0.6$ & as above & $39 \pm 12$ \\
\hline
\end{tabular}

*The concentrations of each substrate before uptake by the assembly were: bisphenol A (BPA), $0.85 \mathrm{mM}$; azulene, $0.16 \mathrm{mM}$; trinitrotoluene (TNT), $0.5 \mathrm{mM}$; Research Department Formula X (RDX), $0.2 \mathrm{mM}$; para-dichlorobenzene ( $p$-DCB), $0.7 \mathrm{mM}$; Alachlor, $0.9 \mathrm{mM}$. For entries 8 and 9 , mixtures of $0.5 \mathrm{mM} \mathrm{BPA}$ and $0.5 \mathrm{mM}$ TNT were used. With the exception of $p$-xylene, reactions were carried out for $24 \mathrm{~h}$ at $25^{\circ} \mathrm{C}$, without stirring; 'reactions were performed with $1.5 \mathrm{ml}$ aqueous solutions of $30 \mathrm{pM}$ PEG-S-capped assemblies; ${ }^{t}$ reactions were performed with $1.1 \mathrm{ml}$ aqueous solutions of 8 pM PEG-S-capped assemblies; ${ }^{s}$ reactions were performed with $61 \mathrm{pM} 50 \%$ PEG-S-50\% hex-S-capped assemblies; "reaction was performed with $50 \mu \mathrm{l}$ p-xylene and $1.5 \mathrm{ml}$ aqueous solution of $61 \mathrm{pM} 50 \%$ PEG-S-50\% hex-S-capped assemblies at $70^{\circ} \mathrm{C}$; ${ }^{\circ}$ reactions were performed with $61 \mathrm{pM}$ PEG-S-capped assemblies.

For entries 3-9 the concentration of substrate molecules in each assembly was calculated from the ratio of thiolate:substrate ${ }^{1} \mathrm{H}-\mathrm{NMR}$ signal intensities after dissolution of the guest-occupied assemblies in $\mathrm{CD}_{2} \mathrm{Cl}_{2}$ (see Supplementary Figs 16-22).

The volumes of these hydrophobic cavities were estimated on the basis of an average nanoparticle radius of $3 \mathrm{~nm}$, a value that includes both the $4 \mathrm{~nm}$ diameter gold core and the hexanethiolate ligands (see Supplementary Fig. 24 and related discussion). The estimated average volumes of individual $O_{\mathrm{h}^{-}}$and $T_{\mathrm{d}}$-symmetry cavities are 28,900 and $5,600 \AA^{3}$, respectively. Given the $223 \AA^{3}$ van der Waal's volume ${ }^{30}$ of BPA, and $55 \%$ by-volume occupancy ${ }^{31}$, the $O_{\mathrm{h}^{-}}$and $T_{\mathrm{d}}$-symmetry cavities should be capable of hosting 71 and 14 molecules, respectively, of this hydrophobic guest. This general situation is illustrated for an $O_{\mathrm{h}}$-symmetry cavity in Fig. $5 \mathrm{c}$. When the numbers of guests per cavity are multiplied by $N O_{\mathrm{h}}$ and $2 N T_{\mathrm{d}}$ holes (where $N=27,400 \mathrm{Au} \mathrm{NPs}$ ), each suprasphere could reasonably accommodate 2.7 million guests (the limit dictated by the $55 \%$ occupancy rule) ${ }^{31}$. It is therefore entirely reasonable that the $\mathrm{O}_{\mathrm{h}^{-}}$and $T_{\mathrm{d}}$-symmetry cavities of a single $200 \mathrm{~nm}$ diameter suprasphere host the observed 2.1 million BPA guests. Similar conclusions are reached for the other molecular guests listed in Table 1, all of which occupy less than $55 \%$ of the total available void space.

For most of the guests listed in Table 1, compliance with the 55\% rule requires that the hydrophobic guests populate void spaces that are buried deeply within the supraspheres, suggestive of an internal architecture that features effective pathways for the extensive diffusion of guest molecules within each assembly. This is explained by the presence of $1.4 \mathrm{~nm}$ pores in the triangular faces of the $3 \mathrm{~N}$ holes. More specifically, the triangular faces of the $\mathrm{O}_{\mathrm{h}}-$ and $T_{\mathrm{d}}$-symmetry cavities (eight and four faces, respectively) each comprise three coplanar Au NPs (Fig. 5d). In each face, the coplanar NPs form an approximately triangular pore that, in the threedimensional structure of the suprasphere, links the $O_{\mathrm{h}}$ and $T_{\mathrm{d}}$ holes. For an average $6 \mathrm{~nm}$ diameter hex-S-protected $\mathrm{Au} \mathrm{NP}$, the height of the pore in each face (labelled $h$ in Fig. $5 \mathrm{~d}$ ) is $1.4 \mathrm{~nm}$. 

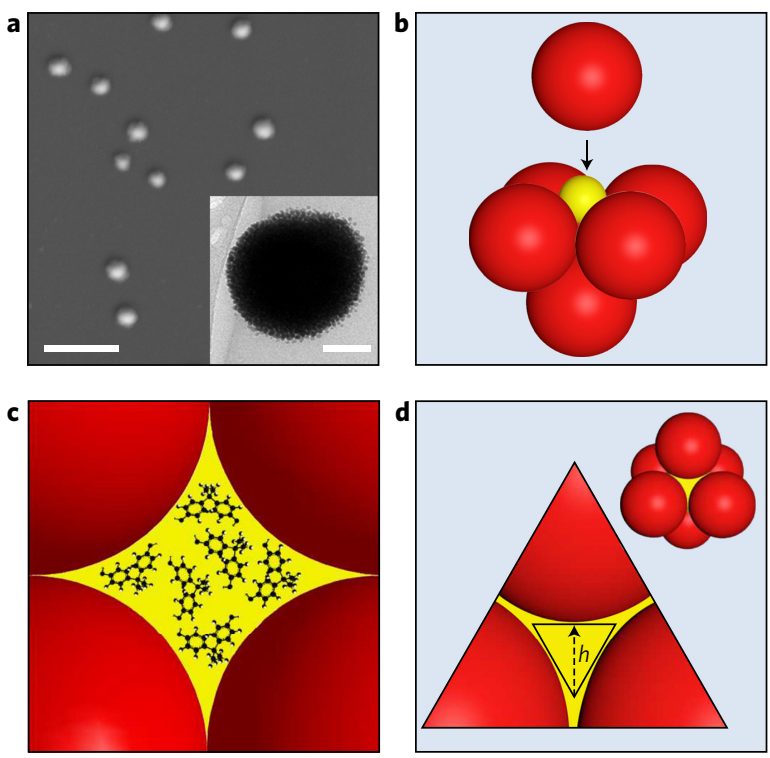

Figure 5 | The hydrophobic cavities and interior pores of the colloidal supraspheres. a, Scanning electron microscope image of dried $200 \mathrm{~nm}$ (average) diameter PEG-S-capped supraspheres (scale bar, $1 \mu \mathrm{m}$ ). Inset: cryo-TEM image of a PEG-S-capped colloidal suprasphere (in this case, approximately $150 \mathrm{~nm}$ in diameter) in its 'native' solution state in vitrified water (scale bar, $50 \mathrm{~nm}$ ). b. Formation of an octahedral $\left(O_{\mathrm{h}}\right)$ hole by closest-packed spheres. For uniform hexanethiolate-capped Au NPs with diameters of $6 \mathrm{~nm}$ (including the thiolate ligands), $2.5 \mathrm{~nm}$ diameter spheres (yellow ball in the figure) fit precisely inside the $O_{\mathrm{h}}$ holes (the total volume of the cavity is nearly four times larger than that of the yellow sphere; see text for details). c, Illustration (drawn to scale) of an $\mathrm{O}_{\mathrm{h}}$-symmetry hole hosting approximately $1 \mathrm{~nm}$ BPA guests. Based on the van der Waals volume $^{30}$ of BPA $\left(223 \AA^{3}\right)$ and the $55 \%$ occupancy rule ${ }^{31}$, each $\mathrm{O}_{\mathrm{h}}$ hole could host 71 BPA guests (see the text for more details). d, Illustration showing a pore (in yellow), with a height, $h$, of $1.4 \mathrm{~nm}$, at the intersection of three coplanar Au NPs that form the faces of $O_{\mathrm{h}}$ and $T_{\mathrm{d}}$ holes (the geometric calculation of $h$ is provided as Supplementary Information). Inset: a three-Au-NP face of an $\mathrm{O}_{\mathrm{h}}$ hole.

These pores are large enough ${ }^{32}$ to allow each of the molecular guests listed in Table 1 to diffuse from the bulk solution to $O_{\mathrm{h}}$-and $T_{\mathrm{d}}$-symmetry cavities buried deeply within the interior of the supraspheres. As such, the hydrophobic domain within each suprasphere is best described as a percolated network of hydrophobic holes. This, combined with the hydrophobic driving force, results in the uptake of the large numbers of organic guests reported in Table 1.

\section{Kinetically gated uptake}

Although the data in Table 1 show the spontaneous uptake of hydrophobic guests to be a general principle, interactions with the capping ligand interface at the surface of the supraspheres can present kinetic barriers to the uptake of specific guests. This suggested that rational modification of the ligand shell could lead to the chemoselective uptake of targeted guests. With PEG-S as the capping ligand, for example, no uptake of TNT was observed. As discussed above, this was overcome by introducing a capping ligand shell that comprised 50\% PEG-S and 50\% hex-S (entry 3) to provide (hex-S-domain) channels for more facile transport of TNT into the interior of the supraspheres. The use of the composition of the capping ligand shell to kinetically gate substrate absorption was then explored using 1:1 mixtures of BPA and TNT (entries 8 and 9; Supplementary Figs 21 and 22). When supraspheres with ligands shells composed of both PEG-S and hex-S were used (entry 8), both substrates were absorbed, and in nearly equal amounts. When completely capped by PEG-S, however (entry 9), BPA alone was found inside the supraspheres. Notably, ligand shells are a unique component of metal and metal-oxide nanoparticles that sets them apart from supramolecular structures and porous metal oxides. In the present case, they provide a higherorder function as versatile membranes for the chemoselective separation of different hydrophobic substrates. This kinetically gated uptake, followed by isolation of the guest cargos via centrifugation and dissolution (Fig. 4), could provide a new and chemoselective method for rapidly concentrating, separating and analysing targeted substances, such as toxic residues or traces of explosive materials.

The use of Au NPs as building blocks also renders the host assemblies opaque, and thus able to protect light-sensitive cargos from photodegradation. In a preliminary experiment, for example, the rapid visible-light-induced isomerization of cis- to trans-azobenzene was prevented on uptake by the suprasphere host (Supplementary Fig. 25).

\section{Conclusions}

Water-soluble assemblies of Au NP building blocks are shown to serve as hosts for the spontaneous uptake of hydrophobic guests. The observed uptake of over two million hydrophobic guests by each $200 \mathrm{~nm}$ suprasphere follows as a natural consequence of moving from molecular to nanoscale building units. Namely, instead of individual supramolecular cages or containers, the use of hydrocarbon-coated gold-nanoparticles as building units leads to multi-container assemblies capable of hosting at least five orders of magnitude more hydrophobic guests. The multiple host domains are provided by void spaces between the hydrocarbon shells of the gold-core building units that, combined with interstitial pores, give a percolated network of hydrophobic cavities for the effective diffusion of guests to host domains buried deeply within the suprasphere. As such, the supraspheres may be viewed as soluble analogs of porous solid-state materials. In this context, their $234 \mathrm{~g} \mathrm{l}^{-1}$ capacity for hydrocarbon guests rivals those of zeolites and metal-organic frameworks. Unlike supramolecular containers or solid-state materials, however, the supraspheres feature capping-ligand shells that serves as tunable gates for the chemoselective uptake (concentration) of targeted molecular guests. After separation by centrifugation, they differ from typical solid-state materials in the ease with which their molecular cargos are quantitatively released by dissolution. The porous supraspheres reported here represent a new class of soluble nano-engineered hosts, combining hydrophobic cavities analogous to those of supramolecular containers, with internal architectures and capacities typical of porous solid-state materials. Furthermore, comprising hydrophobic cores, and coated by chemoselective ligand shells, they are capable of unique functions not readily achievable by either class of well-known host structure.

\section{Methods}

Methods and any associated references are available in the online version of the paper.

\section{Received 25 May 2016; accepted 26 September 2016;} published online 14 November 2016; corrected online 16 November 2016

\section{References}

1. Cook, T. R. \& Stang, P. J. Recent developments in the preparation and chemistry of metallacycles and metallacages via coordination. Chem. Rev. 115, 7001-7045 (2015)

2. Brown, C. J., Toste, F. D., Bergman, R. G. \& Raymond, K. N. Supramolecular catalysis in metal-ligand cluster hosts. Chem. Rev. 115, 3012-3035 (2015).

3. Ajami, D. \& Rebek, J. More chemistry in small spaces. Acc. Chem. Res. 46, 990-999 (2013).

4. Sato, S. et al. Fluorous nanodroplets structurally confined in an organopalladium sphere. Science 313, 1273-1276 (2006). 
5. Kopilevich, S., Müller, A. \& Weinstock, I. A. Amplified rate acceleration by simultaneous up-regulation of multiple active sites in an endo-functionalized porous capsule. J. Am. Chem. Soc. 137, 12740-12743 (2015).

6. Chmelik, C. \& Kärger, J. In situ study on molecular diffusion phenomena in nanoporous catalytic solids. Chem. Soc. Rev. 39, 4864-4884 (2010).

7. Yang, S. et al. Supramolecular binding and separation of hydrocarbons within a functionalized porous metal-organic framework. Nat. Chem. 7, 121-129 (2015).

8. Zhao, H. et al. Reversible trapping and reaction acceleration within dynamically self-assembling nanoflasks. Nat. Nanotech. 11, 82-88 (2016).

9. Grego, A., Müller, A. \& Weinstock, I. A. Stepwise-resolved thermodynamics of hydrophobic self-assembly. Angew. Chem. Int. Ed. 52, 8358-8362 (2013).

10. Blokzijl, W. \& Engberts, J. B. F. N. Hydrophobic effects. Opinions and facts. Angew. Chem. Int. Ed. Engl. 32, 1545-1579 (1993).

11. Kopilevich, S., Gottlieb, H., Keinan-Adamsky, K., Müller, A. \& Weinstock, I. A. The uptake and assembly of alkanes within a porous nanocapsule in water: new information about hydrophobic confinement. Angew. Chem. Int. Ed. 55, 4476-4481 (2016).

12. Song, Z. et al. Nanovalved adsorbents for $\mathrm{CH}_{4}$ storage. Nano Lett. 16, 3309-3313 (2016).

13. Mason, J. A. et al. Methane storage in flexible metal-organic frameworks with intrinsic thermal management. Nature 527, 357-361 (2015).

14. Boal, A. K. et al. Self-assembly of nanoparticles into structured spherical and network aggregates. Nature 404, 746-748 (2000).

15. Maye, M. M. et al. Mediator-template assembly of nanoparticles. J. Am. Chem. Soc. 127, 1519-1529 (2005).

16. Klajn, R., Bishop, K. J. M. \& Grzybowski, B. A. Light-controlled self-assembly of reversible and irreversible nanoparticle suprastructures. Proc. Natl Acad. Sci. USA 104, 10305-10309 (2007).

17. Xia, Y. et al. Self-assembly of self-limiting monodisperse supraparticles from polydisperse nanoparticles. Nat. Nanotech. 6, 580-587 (2011).

18. Sánchez-Iglesias, A. et al. Hydrophobic interactions modulate self-assembly of nanoparticles. ACS Nano 6, 11059-11065 (2012).

19. Wang, T., LaMontagne, D., Lynch, J., Zhuang, J. \& Cao, Y. C. Colloidal superparticles from nanoparticle assembly. Chem. Soc. Rev. 42, 2804-2823 (2013)

20. Choueiri, R. M., Klinkova, A., Therien-Aubin, H., Rubinstein, M. \& Kumacheva, E. Structural transitions in nanoparticle assemblies governed by competing nanoscale forces. J. Am. Chem. Soc. 135, 10262-10265 (2013).

21. Wang, J. et al. Nucleation-controlled polymerization of nanoparticles into supramolecular structures. J. Am. Chem. Soc. 135, 11417-11420 (2013).

22. Weinstock, I. A., Cowan, J. J., Barbuzzi, E. M. G., Zeng, H. \& Hill, C. L. Equilibria between $\alpha$ and $\beta$ isomers of keggin heteropolytungstates. J. Am. Chem. Soc. 121, 4608-4617 (1999).

23. Wang, Y. et al. Self-assembly and structure of directly imaged inorganic-anion monolayers on a gold nanoparticle. J. Am. Chem. Soc. 131, 17412-17422 (2009).
24. Wang, Y., Zeiri, O., Sharet, S. \& Weinstock, I. A. Role of the alkali-metal cation size in the self-assembly of polyoxometalate-monolayer shells on gold nanoparticles. Inorg. Chem. 51, 7436-7438 (2012).

25. Wang, Y. \& Weinstock, I. A. Polyoxometalate-decorated nanoparticles. Chem. Soc. Rev. 41, 7479-7496 (2012).

26. Zeiri, O., Wang, Y., Neyman, A., Stellacci, F. \& Weinstock, I. A. Ligand-shelldirected assembly and depolymerization of patchy nanoparticles. Angew. Chem. Int. Ed. 52, 968-972 (2013).

27. Wang, Y., Zeiri, O., Neyman, A., Stellacci, F. \& Weinstock, I. A. Nucleation and island growth of alkanethiolate ligand domains on gold nanoparticles. ACS Nano 6, 629-640 (2012)

28. Wang, Y., Zeiri, O., Meshi, L., Stellacci, F. \& Weinstock, I. A. Regioselective placement of alkanethiolate domains on tetrahedral and octahedral gold nanocrystals. Chem. Commun. 48, 9765-9767 (2012).

29. West, A. R. Solid State Chemistry and its Applications (Wiley, 2014).

30. Zhao, Y. H., Abraham, M. H. \& Zissimos, A. M. Fast calculation of van der waals volume as a sum of atomic and bond contributions and its application to drug compounds. J. Org. Chem. 68, 7368-7373 (2003).

31. Mecozzi, S. \& Rebek, J. J. Jr The $55 \%$ solution: a formula for molecular recognition in the liquid state. Chem. Eur. J. 4, 1016-1022 (1998).

32. Ziv, A. et al. Flexible pores of a metal oxide-based capsule permit entry of comparatively larger organic guests. J. Am. Chem. Soc. 131, 6380-6382 (2009).

\section{Acknowledgements}

I.A.W thanks the Israel Science Foundation (190/13) and I.A.W. and F.S. thank the US Israel Binational Science Foundation (2008277) for support. We thank E. Gadot for TEM imaging and I. Willner for samples of TNT and RDX

\section{Author contributions}

Y.W. and O.Z. contributed equally to this work. I.A.W. initiated and directed the research, O.Z., Y.W., B.L.O., F.S. and I.A.W. designed the experiments, O.Z., Y.W., M.R. and B.L.O. carried out the experimental work, and O.Z., Y.W., B.L.O., F.S. and I.A.W. interpreted the experimental data. I.A.W. coordinated the writing of the manuscript with input from all authors.

\section{Additional information}

Supplementary information is available in the online version of the paper. Reprints and permissions information is available online at www.nature.com/reprints. Correspondence and requests for materials should be addressed to I.A.W.

\section{Competing financial interests}

The authors declare no competing financial interests. 


\section{Methods}

Synthesis of colloidal supraspheres. Citrate-stabilized $4 \mathrm{~nm}$ diameter Au NPs, and ligand exchange of citrate by 1 , were performed according to published methods ${ }^{26}$. The solubility of hex-SH in water $(0.361 \mathrm{mM}$ at pH 7 and $298 \mathrm{~K})$ is much larger than that needed to form complete monolayer shells on the surfaces of typical aqueous solutions of $4 \mathrm{~nm}$ Au NPs. Stock solutions of hex-SH were therefore prepared by adding $500 \mu \mathrm{l}$ of pure hex-SH to $10 \mathrm{ml}$ of water in a $20 \mathrm{ml}$ vial, giving a two-phase system. After $12 \mathrm{~h}$, the aqueous phase (the lower layer) was transferred by glass pipette to a new $20 \mathrm{ml}$ vial. DLS confirmed the absence of hex-SH aggregates in these dilute solutions. Owing to the adsorption of hexanethiol on glassware and plastic pipette tips, however, concentrations of hex-SH in these stock solutions (determined by standard methods) ${ }^{33}$ consistently deviated from the analytical values used in their preparation, even when kept under inert atmospheres. To overcome this, the SPR of the Au NPs themselves was used as a quantitative spectrophotometric tool for monitoring the assembly process. For this, aliquots of the hex-SH solution were added to a $10 \mathrm{ml}$ solution of the 1-protected $4 \mathrm{~nm}$ Au NPs in a $20 \mathrm{ml}$ vial, with vigorous magnetic stirring (using a $1 \mathrm{~cm}$ long Teflon-coated magnet) for approximately $4 \mathrm{~min}$ after each addition. The reaction was monitored by ultravioletvisible spectroscopy to identify the decrease in changes in the SPR absorbance at $535 \mathrm{~nm}$ (indicative of the displacement of 1 by hex-SH) and the increase in absorbance at $750 \mathrm{~nm}$ (indicative of nanoparticle aggregation). Stabilization of the fully formed supraspheres was achieved by then adding volumes of freshly prepared $1.5 \mathrm{mM} \mathrm{HSR}^{-}$, PEG-SH or HSR ${ }^{+}$solutions (typically around $30 \mu \mathrm{l}$ ), as needed to displace the final few per cent of 1 remaining on the outer surfaces of the supraspheres, shaken gently by hand for approximately $30 \mathrm{~s}$, and then set aside for $12 \mathrm{~h}$. The $50 \%$ PEG-S-capped:50\% hex-S-capped supraspheres were synthesized by capping freshly assembled supraspheres with $50 \%$ of freshly prepared $1.5 \mathrm{mM}$ PEG-S (approximately $15 \mu \mathrm{l}$ ), shaking gently and after $12 \mathrm{~h}$ adding $50 \%$ of freshly prepared $1.5 \mathrm{mM}$ hex-SH in DMF (approximately $15 \mu \mathrm{l}$ ), followed by $20 \mathrm{~min}$ of gentle mechanical stirring.

Guest uptake and release. The following (typical) procedure was used for BPA and other relatively non-volatile guests. After synthesis, the PEG-S-capped supraspheres were isolated by centrifugation ( $15 \mathrm{~min}$ at 7,000 r.p.m.), dissolved in water and 'spun down' three times (each time for $20 \mathrm{~min}$ at 8,000 r.p.m.). The pellet was then added to $1.5 \mathrm{ml}$ of aqueous BPA $\left(0.193 \mathrm{mg} \mathrm{ml}^{-1}, 0.85 \mathrm{mM}\right)$ in a $4 \mathrm{ml}$ glass vial. After $24 \mathrm{~h}$ at room temperature, the supraspheres were completely removed from the solution by centrifugation (10 $\mathrm{min}$ at 13,500 r.p.m.), and the concentration of BPA in the supernatant was determined by ultraviolet-visible spectroscopy. The pellet was then washed with $1.5 \mathrm{ml}$ of water, isolated by centrifugation (10 $\mathrm{min}$ at 13,500 r.p.m.) and the final $20 \mu \mathrm{l}$ pellet containing BPA guests was added to $0.5 \mathrm{ml} \mathrm{CD} \mathrm{Cl}_{2}$ and stirred for $1 \mathrm{~h}$ at room temperature. The released bisphenol A was quantified by ${ }^{1} \mathrm{H}$ NMR spectroscopy as described in the text. In the above method, solid samples of the organic guests were dissolved in water to give dilute aqueous solutions, which were then combined with the supraspheres. For dilute solutions of $p$-xylene (entry 7), uptake after $24 \mathrm{~h}$ at room temperature was too small to quantify by ${ }^{1} \mathrm{H}$ NMR spectroscopy. Unlike the other guests (which are solids at room temperature), $p$-xylene is a somewhat volatile liquid and formed a film on the glass walls above the solution in the reaction vial. To overcome this, a layer of $p$-xylene $(50 \mu \mathrm{l})$ was placed on the aqueous solution, after which the vial was sealed and warmed to $70{ }^{\circ} \mathrm{C}$ for $24 \mathrm{~h}$. After separation of the supraspheres, dissolution in $\mathrm{CD}_{2} \mathrm{Cl}_{2}$ revealed the uptake of 1.6 million molecules of $p$-xylene.

Guest occupancy and Rebek's 55\% rule. The total void space formed by approximately $O_{\mathrm{h}}$ and $T_{\mathrm{d}}$ cavities must be at least $26 \%$ of the volume of each supraspherical assembly. This (ideal) $26 \%$ void space is much larger than that needed to accommodate the numerous guests reported in Table 1. Each $200 \mathrm{~nm}$ diameter suprasphere has a total volume of $4.2 \times 10^{6} \mathrm{~nm}^{3}$, giving a $26 \%$ void space of $1.1 \times 10^{6} \mathrm{~nm}^{3}$. The percentage of this void space occupied by hydrophobic guests was evaluated based on the van der Waals volumes ${ }^{30}$ of the encapsulated organic molecules (see Table 1 and calculations in the Supplementary Information). The van der Waals volume of bisphenol A, for example, is estimated at $223 \AA^{3}\left(\right.$ or $0.223 \mathrm{~nm}^{3}$ ), such that 2.1 million of these guests occupy a volume of $4.7 \times 10^{5} \mathrm{~nm}^{3}$, corresponding to $43 \%$ of the void space within each suprasphere (far-right column in Table 1 ). Importantly, this $43 \%$ value, as well as corresponding values for the other molecules listed in Table 1, complies with Rebek's $55 \%$ rule $^{31}$ for occupancy of host cavities by hydrophobic guests.

\section{References}

33. Dalman, G. \& Gorin, G. Ionization constant of hexanethiol from solubility measurements. J. Org. Chem. 26, 4682-4684 (1961). 